\title{
Produção e qualidade de óleos essenciais de Mentha arvensis em resposta à inoculação de fungos micorrízicos arbusculares
}

\author{
Marta Simone Mendonça Freitas ${ }^{(1)}$, Marco Antonio Martins ${ }^{(1)}$ e Ivo José Curcino Vieira(1)
}

(1)Universidade Estadual do Norte Fluminense, Centro de Ciências e Tecnologias Agropecuárias, CEP 28013-600 Campos dos Goytacazes, RJ.E-mail:msimone@uenf.br, marco@uenf.br, curcino@uenf.br

Resumo - O objetivo deste trabalho foi avaliar os efeitos de diferentes espécies de fungos micorrízicos arbusculares (FMA) na produção e qualidade de óleos essenciais de Mentha arvensis L., cultivada com diferentes doses de fósforo. O trabalho foi realizado em casa de vegetação e o delineamento estatístico utilizado foi de blocos ao acaso com 20 tratamentos, constituídos pela combinação de cinco tratamentos microbiológicos (Glomus clarum Nicolson \& Schenck, Glomus etunicatum Becker \& Gerdemann, Gigaspora margarita Becker \& Hall, Acaulospora scrobiculata Trappe e controle) e quatro doses de $\mathrm{P}\left(0,50,100\right.$ e $\left.200 \mathrm{mg} \mathrm{kg}^{-1}\right)$, com quatro repetições. As plantas foram colhidas 65 dias após o plantio. Sem adubação fosfatada, os fungos Gigaspora margarita e Glomus clarum aumentaram em 206 e 198\%, respectivamente, a produção de matéria fresca em relação ao tratamento controle. A maior produção de matéria fresca ( $90 \mathrm{~g}$ por vaso) foi observada no tratamento de A. scrobiculata com $133 \mathrm{mg} \mathrm{kg}^{-1}$ de fósforo. Na ausência de adubação fosfatada, teores de óleos essenciais e de mentol no óleo foram menores em plantas sem inoculação, e os tratamentos com inoculação proporcionaram incrementos de até $89 \%$ nos teores de óleos e de mentol, em relação ao tratamento sem inoculação. Com o incremento da adubação fosfatada, não foram observados incrementos dos teores de óleos essenciais e mentol em razão dos FMA. As

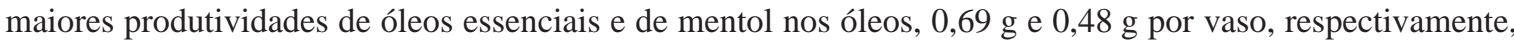
foram encontradas em plantas com inoculação de Acaulospora scrobiculata nas doses de P de $126 \mathrm{e} 123 \mathrm{mg} \mathrm{kg}^{-1} \mathrm{de}^{-}$ solo, respectivamente.

Termos para indexação: mentol, micorriza, fósforo, planta medicinal e aromática.

\section{Yield and Quality of essential oils of Mentha arvensis in response to inoculation with arbuscular mycorrhizal fungi}

Abstract - The objective of this work was to evaluate the effects of different species of arbuscular mycorrhizal fungi on the vegetative growth, production and composition of essential oil of Mentha arvensis L., grown in different phosphorus levels. The work was carried out under greenhouse conditions and a randomized block design was used with 20 treatments, constituted by the combination of five microbiological treatments (Glomus clarum Nicolson \& Schenck, Glomus etunicatum Becker \& Gerdemann, Gigaspora margarita Becker \& Hall, Acaulospora scrobiculata Trappe and control) and four P levels $\left(0,50,100,200 \mathrm{mg} \mathrm{kg}^{-1}\right)$, with four repetitions. Plants were harvested 65 days after transplanting. Results showed that, when no phosphorus was added, Gigaspora margarita and Glomus clarum increased, in 206\% and 198\%, respectively, the fresh matter production of shoots, compared to the control treatment. The highest fresh matter production $(90 \mathrm{~g}$ por pot) was observed in plants inoculated with $A$. scrobiculata at $133 \mathrm{mg} \mathrm{kg}^{-1}$ of phosphorus. When phosphorus was not used, the essential oil content and menthol levels in the oil were smaller in plants not inoculated, and the inoculated treatments provided increments of up to $89 \%$ in the essential oil and menthol contents in relation to treatment not inoculated. No increment in essential oil and menthol contents occurred when the doses of $\mathrm{P}$ were increased. The highest essential oil and menthol production, $0.69 \mathrm{~g}$ and $0.48 \mathrm{~g}$ for pot, respectively, were found in plants inoculated with A. scrobiculata at the P levels and 126 and $123 \mathrm{mg}$ for $\mathrm{kg}$ of soil, respectively.

Index terms: menthol, mycorrhiza, phosphorus, aromatic and medicinal plants.

\section{Introdução}

Entre as plantas medicinais e aromáticas, a Mentha arvensis L. é uma espécie de interesse econômico, porque os seus óleos essenciais são uma rica fonte de mentol, com várias aplicações industriais, como em produtos de higiene bucal, flavorizantes, aromatizantes de alimentos e bebidas, em perfumaria e produtos farmacêuticos (Matos, 2000; Kumar et al., 2002). 
A biossíntese dos metabólitos secundários e, conseqüentemente, dos princípios ativos em plantas medici nais e aromáticas depende de fatores genéticos, fisiológicos e ambientais. Assim, os fatores que influenciam as variações nas concentrações destes princípios em plantas deve ser avaliado, visando obter uma matériaprima de melhor qualidade, já que a qualidade das plantas medicinais está relacionada ao seu teor de princípios ativos e, portanto, à sua eficácia terapêutica. Entre os fatores ambientais, a inoculação de fungos micorrízicos arbusculares (FMA) em plantas medicinais e aromáticas pode influenciar na produção de princípios ativos (Moreira \& Siqueira, 2002; Kapoor et al., 2002b), seja por efeito de melhor estado nutricional ou como resposta de defesa do hospedeiro à presença do fungo (Volpin et al., 1994).

Os efeitos benéficos dos FMA têm sido demonstrados em variadas condições e espécies vegetais, estimulando o crescimento vegetal como uma consequiência de seu efeito na nutrição mineral da planta, principalmente no aumento da absorção de fósforo (Smith \& Read, 1997; Bressan et al., 2001; Pralon \& Martins, 2001).

Trabalhos com plantas medicinais e aromáticas, avaliando a ocorrência dos FMA, foram realizados por $\mathrm{Fa}$ ria et al. (2000) e Grotkass et al. (2000). Entretanto, pouco se conhece sobre a influência destes fungos no teor e na composição dos óleos essenciais da Mentha.

O objetivo deste trabalho foi avaliar os efeitos de diferentes fungos micorrízicos arbusculares na produção e qualidade de óleos essenciais de Mentha arvensis L., cultivada em diferentes doses de fósforo.

\section{Material e Métodos}

O experimento foi realizado em casa de vegetação no Campus da Universidade Estadual do Norte Fluminense Darcy Ribeiro, localizada no Município de Campos dos Goytacazes, RJ (Latitude = 21 ${ }^{\circ} 19^{\prime} 23 "$; Longitude $=41^{\circ} 10^{\prime} 40^{\prime \prime} \mathrm{W}$; Altitude $=14 \mathrm{~m}$ ), de 18/03/ 2002 a 22/05/2002. Durante o período experimental, as temperaturas diárias máximas variaram de 41,3 a $28,6^{\circ} \mathrm{C}$, com média de $35,4^{\circ} \mathrm{C}$ e as temperaturas diárias mínimas estiveram entre $24,1 \mathrm{e} 16,1^{\circ} \mathrm{C}$, com média de $20,3^{\circ} \mathrm{C}$ A umidade relativa máxima teve média de 93,3\%, com variações entre 97,6 e $89,8 \%$ e a umidade relativa mínima com média de $28,9 \%$, variou entre 44 e $16,7 \%$.

O delineamento experimental foi de blocos casualizados em arranjo fatorial $5 \times 4$, sendo cinco trata- mentos (Glomus clarum Nicolson \& Schenck, Glomus etunicatum Becker \& Gerdemann, Gigaspora margarita Becker \& Hall, Acaulospora scrobiculata Trappe e controle) e quatro doses de P $(0,50,100$ e $200 \mathrm{mg} \mathrm{kg}^{-1}$ ), com quatro repetições. A unidade experimental foi composta por um vaso de plástico contendo $3 \mathrm{~L}$ de solo e duas plantas por vaso.

O substrato utilizado era composto de amostras (0-20 cm) coletadas de um Latossolo Amarelo distrófico, na Escola Estadual Agrícola Antônio Sarlo, Campos dos Goytacazes, RJ. O solo coletado foi peneirado e posteriormente esterilizado em autoclave por duas vezes, a $121^{\circ} \mathrm{C}$ por 1 hora, com a finalidade de eliminação dos FMA nativos. Após a autoclavagem, o substrato apresentou as seguintes características químicas e granulométricas: $\mathrm{pH}$ em água, 5,2; matéria orgânica, $18,8 \mathrm{~g} \mathrm{dm}^{-3} ; \mathrm{P}, 6 \mathrm{mg} \mathrm{dm}{ }^{-3} ; \mathrm{S}, 29,8 \mathrm{mg} \mathrm{dm}^{-3} ; \mathrm{K}^{+}$, $64 \mathrm{mg} \mathrm{dm}{ }^{-3} ; \mathrm{Ca}^{2+}, 1,6 \mathrm{cmol}_{\mathrm{c}} \mathrm{dm}^{-3} ; \mathrm{Mg}^{2+}, 1,0 \mathrm{cmol}_{\mathrm{c}} \mathrm{dm}^{-3}$; $\mathrm{Al}^{3+}, 0,2 \mathrm{cmol}_{\mathrm{c}} \mathrm{dm}^{-3} ; \mathrm{H}+\mathrm{Al}, 3,5 \mathrm{cmol}_{\mathrm{c}} \mathrm{dm}^{-3} ; \mathrm{SB}, 2,8 \mathrm{cmol}_{\mathrm{c}}$ $\mathrm{dm}^{-3} ; \mathrm{T}, 6,3 \mathrm{cmol}_{\mathrm{c}} \mathrm{dm}^{-3} ; \mathrm{Fe}, 65 \mathrm{mg} \mathrm{dm}^{-3} ; \mathrm{Cu}, 0,2 \mathrm{mg} \mathrm{dm}^{-3}$; $\mathrm{Zn}, 1,7 \mathrm{mg} \mathrm{dm}^{-3}$; Mn, 11,2 $\mathrm{mg} \mathrm{dm}^{-3}$; B, 0,58 $\mathrm{mg} \mathrm{dm}^{-3}$; areia, $560 \mathrm{~g} \mathrm{~kg}^{-1}$; silte, $60 \mathrm{~g} \mathrm{~kg}^{-1} \mathrm{e}$ argila, $380 \mathrm{~g} \mathrm{~kg}^{-1}$. A dose de $\mathrm{k} \mathrm{em}$ todos os tratamentos foi de $127 \mathrm{mg} \mathrm{kg}^{-1}$ de solo, utilizado como fonte o $\mathrm{K}_{2} \mathrm{SO}_{4} \mathrm{e} \mathrm{KH}_{2} \mathrm{PO}_{4}$. A dose de $\mathrm{N}$ foi, em todos os tratamentos, de $20 \mathrm{mg} \mathrm{kg}^{-1}$ de solo, na forma de $\mathrm{NH}_{4} \mathrm{NO}_{3}$ e a dose de cálcio foi de $4 \mathrm{cmol}_{\mathrm{c}} \mathrm{kg}^{-1}$, utilizando como fonte $\mathrm{CaCO}_{3}$. As fontes de P utilizadas foram $\mathrm{KH}_{2} \mathrm{PO}_{4} \mathrm{e} \mathrm{NaH} \mathrm{NO}_{4}$. Após a aplicação das doses de $\mathrm{P}\left(0,50,100\right.$ e $200 \mathrm{mg} \mathrm{kg}^{-1}$ de solo), os vasos permaneceram umedecidos e incubados por 45 dias, onde posteriormente foram realizadas extrações de $\mathrm{P}$ (Mehlich-1), obtendo-se 6, 27, 53 e $121 \mathrm{mg} \mathrm{dm}^{-3}$ de P, respectivamente, em relação às doses de $0,50,100 \mathrm{e}$ $200 \mathrm{mg} \mathrm{kg}^{-1}$

O substrato utilizado na multiplicação do inóculo foi uma mistura de solo e areia na proporção de 1:2 (v/v), esterilizado em autoclave por duas vezes, a $121^{\circ} \mathrm{C}$, por uma hora. Ao substrato, colocado em vasos com capacidade de $3 \mathrm{~L}$, foram adicionados $50 \mathrm{~g}$ de inóculo inicial, constituindo uma mistura de solo contendo esporos, hifas e raízes colonizadas, com o fungo de cada espécie a ser estudada: Glomus clarum, Glomus etunicatum, Gigaspora margarita e Acaulospora scrobiculata. $\mathrm{O}$ inóculo inicial dos fungos foi retirado da coleção do Setor de Microbiologia do Solo da UENF. A seguir, 15 sementes de Brachiaria brizantha, desinfestadas com solução $0,5 \%$ de hipoclorito de sódio durante 10 min e lavadas com água deionizada, foram semeadas em cada vaso. Quatro meses após a semeadura, a parte aérea foi 
cortada e trinta dias após o corte, a mistura de solo contendo esporos $(20,23,17$ e 18 esporos por g de solo das espécies Glomus clarum, Glomus etunicatum, Gigaspora margarita e Acaulospora scrobiculata, respectivamente), hifas e raízes finas cortadas, foi utilizada como inóculo.

Estacas herbáceas de aproximadamente $10 \mathrm{~cm}$ de comprimento, com dois nós e dois pares de folhas foram coletadas no horto de plantas medicinais, localizado no Município de Vargem Alta, ES. O materia propagativo foi plantado, enterrando um dos nós no substrato, em bandejas de isopor de 72 células com capacidade para $100 \mathrm{~mL}$ de substrato/célula. O substrato utilizado foi areia autoclavada como descrito anteriormente. As bandejas foram colocadas em casa de vegetação, com nebulização de duas em duas horas, por 20 dias, até o enraizamento das estacas. Ao final desse período foram selecionadas 160 estacas com uniformidade no crescimento. A seguir, foram transplantada duas estacas por vaso e adicionados $50 \mathrm{~g}$ do inóculo das espécies Glomus clarum, Glomus etunicatum, Gigaspora margarita e Acaulospora scrobiculata, deixando-se o tratamento controle sem inoculação. Além da inoculação, cada vaso recebeu $10 \mathrm{~mL}$ de uma suspensão de solo obtida a partir de $50 \mathrm{~mL}$ de solo de cada isolado utilizado, que foi peneirado e filtrado em papelfiltro para a eliminação de propágulos de FMA com o objetivo de equilibrar a microbiota entre as amostras. $\mathrm{O}$ tratamento controle não recebeu o inóculo, mas solução de $10 \mathrm{~mL}$ da suspensão de solo. Os inóculos foram colocados em contato com as raízes. As plantas foram irrigadas diariamente, utilizando-se água deionizada, de modo a manter o solo úmido, e sem encharcamento, durante o período experimental.

As plantas foram coletadas 65 dias após o transplantio nos vasos, período em que se iniciava a fase de floração. Foram quantificadas a massa da matéria fresca da parte aérea, a porcentagem de colonização micorrízica, o teor de óleos essenciais na matéria fresca e de mentol nos óleos essenciais. A pesagem da matéria fresca foi realizada dentro da casa de vegetação, utilizando uma balança digital com escala em grama e precisão de duas casas decimais. A determinação da porcentagem de colonização micorrízica foi realizada pelo método da interseção em placa de Petri quadriculada (Giovannetti \& Mosse, 1980), após a coloração das raízes em azul de metil (Grace \& Stribley, 1991).
Na extração e determinação do teor de óleos essenciais, as plantas foram coletadas no horário de 7 às $8 \mathrm{~h}$ da manhã, identificadas e armazenadas em freezer a $-18^{\circ} \mathrm{C}$. A extração foi feita mediante destilação por arraste a vapor em aparelho tipo Clevenger, modelo MA 553, durante uma hora (Maia, 1998). O teor dos óleos foi determinado pela massa dos óleos em balança analítica, e expresso em porcentagem massa/massa (g de óleos por $100 \mathrm{~g}$ de matéria fresca).

A análise química dos óleos essenciais foi realizada por cromatografia gasosa, com coluna capilar DB-1 (25 m x $0,25 \mathrm{~mm}$ ), rampa de temperatura (temperatura inicial $50^{\circ} \mathrm{C}$ e final $230^{\circ} \mathrm{C}$ ) com uma taxa de $15^{\circ} \mathrm{C} \mathrm{min}^{-1}$, temperatura do injetor a $200^{\circ} \mathrm{C}$ e do detector a $230^{\circ} \mathrm{C}$, hélio utilizado como gás de arraste e fluxo de $0,6 \mathrm{~mL} \mathrm{~min}^{-1}$. Na quantificação do mentol, foi utilizado o método de calibração externa. Foram preparadas soluções do padrão de (-)mentol, marca Sigma ${ }^{\circledR}$, com concentrações de 0,$1 ; 0,3 ; 0,5 ; 0,7$ e $1,0 \mathrm{mg} \mathrm{mL}^{-1}$. O padrão foi diluído em clorofórmio e injetado $(1 \mu \mathrm{L})$ no cromatógrafo gasoso, obtendo-se o cromatograma correspondente a cada uma das concentrações.

Os dados foram submetidos à análise de variância. Para os dados quantitativos, como o fator dose de P, foi utilizada análise de regressão polinomial, teste $\mathrm{F}$ da análise de variância da regressão e coeficientes do modelo estatisticamente significativo e maior $\mathrm{R}^{2}$. Para os dados qualitativos, no caso do fator tratamentos microbiológicos, foi utilizado o teste de Tukey a 5\% de probabilidade.

\section{Resultados e Discussão}

Houve interação dos FMA e doses de $\mathrm{P}$ em relação à variável massa de matéria fresca (Figura 1). $\mathrm{Na}$ ausência da adubação fosfatada, as maiores produções de matéria fresca da parte aérea da Mentha arvensis L. foram obtidas nos tratamentos com os fungos Glomus clarum e Gigaspora margarita, que aumentaram 207 e 198\%, respectivamente, em relação ao tratamento controle. As espécies Glomus etunicatum e Acaulospora scrobiculata foram menos eficientes em promover o crescimento das plantas em relação às outras espécies. Entretanto, a maior produtividade de matéria fresca da parte aérea, $90 \mathrm{~g}$ por vaso, foi estimada pelo ponto máximo da curva, quando as plantas foram submetidas à inoculação de Acaulospora scrobiculata e adubadas com $133 \mathrm{mg}$ de $\mathrm{P}$ por $\mathrm{kg}$ de solo (Figura 1). 
Vários trabalhos relatam o beneficio dos FMA em condições de baixa disponibilidade de nutrientes no solo, principalmente fósforo (Martins et al., 2000; Soares \& Martins, 2000; Jackson et al., 2002). Com relação ao aumento na produção vegetal, resultados semelhantes aos encontrados com Glomus clarum e Gigaspora margarita, na dose 0 de $\mathrm{P}$, foram observados por Samarão \& Martins (1999), em mudas de goiabeira em Campos dos Goytacazes, RJ. Gupta et al. (2002), com diferentes cultivares de Mentha arvensis L. e a espécie de fungo Glomus fasciculatum, constataram incrementos significativos na produção de matéria fresca em campo, com média entre as cultivares de $62 \%$ de raízes colonizadas, em solo com $17 \mathrm{mg}$ de $\mathrm{P}$ por kg.

Em relação à porcentagem de colonização micorrízica, houve interação entre as espécies de fungo e as doses de P, e as espécies de FMA apresentaram comportamento diferenciado, de acordo com a disponibilidade de P no solo (Figura 2). Nos tratamentos com Glomus clarum e Gigaspora margarita, houve um decréscimo de 81 para $40 \%$ e de 69 para $38 \%$, respec tivamente, na porcentagem de colonização micorrízica com o incremento de P no solo. Samarão \& Martin (1999) verificaram que, em mudas de goiabeira, a porcentagem de colonização micorrízica foi reduzida de $86 \%$ para $52 \%$ e de $85 \%$ para $66 \%$, quando foram submetidas à inoculação de Glomus clarum e Gigaspora margarita, respectivamente. Ou seja, a simbiose com esses fungos foi menos eficiente em promover o crescimento das mudas, com uma dose de $50 \mathrm{mg} \mathrm{kg}^{-1}$ de fósforo. $\mathrm{O}$ efeito negativo no incremento de $\mathrm{P}$ na porcentagem de raízes colonizadas também foi observado por Nogueira \& Cardoso (2000) e Costa et al. (2001), quando foram utilizadas as espécies de FMA citadas.

Moreira \& Siqueira (2002) relataram que, em solo muito deficiente em $\mathrm{P}$, a aplicação de pequena quantidade desse elemento favorece a colonização e a esporulação dos FMA. Tanto a colonização como a esporulação dos FMA são inibidas em doses elevadas de $\mathrm{P}$ e o efeito da adição depende da espécie vegetal. As espécies Glomus etunicatum e Acaulospora scrobiculata só apresentaram benefícios para a planta hospedeira quando foi adicionado fósforo. Com a Acaulospora scrobiculata, na dose de $133 \mathrm{mg} \mathrm{kg}^{-1}$, a maior produção de matéria fresca foi estimada em $90 \mathrm{~g}$ por vaso (Figura 1). Bressan \& Vasconcellos (2002), testando doses de $0,50,100$ e $200 \mathrm{mg}$ de $\mathrm{P}$ por $\mathrm{kg}$ de solo e a espécie de fungo Glomus etunicatum em milho, observaram que a porcentagem de colonização aumentou até a dose de $124 \mathrm{mg} \mathrm{kg}^{-1}$, havendo uma queda

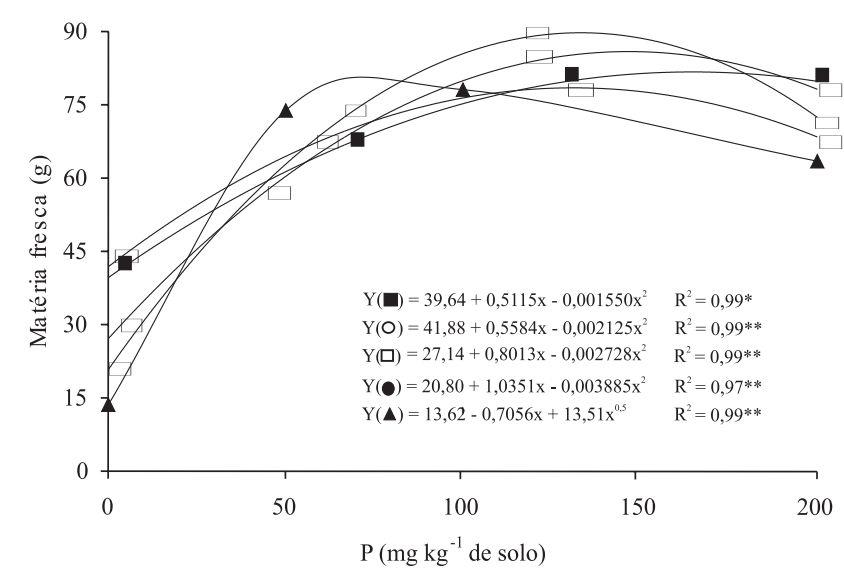

Figura 1. Massa de matéria fresca da parte aérea de Mentha arvensis $\mathrm{L}$. em razão das doses de $\mathrm{P}$ e dos fungos micorrízicos arbusculares Gigaspora margarita (ם), Glomus clarum (O), Glomus etunicatum $(\square)$, Acaulospora scrobiculata $(\bullet)$ e controle $(\mathbf{\Lambda})$.

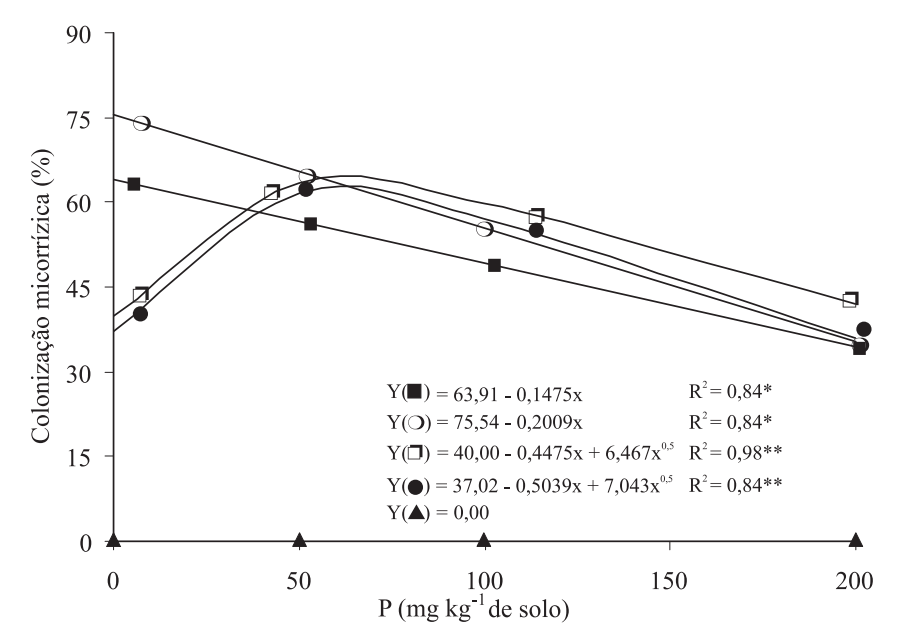

Figura 2. Colonização micorrízica em raízes de Mentha arvensis L. em razão da doses de $\mathrm{P}$ e dos fungos micorrízicos arbusculares Gigaspora margarita (ם), Glomus clarum (O), Glomus etunicatum $(\square)$, Acaulospora scrobiculata $(\bullet)$ e controle $(\mathbf{\Delta})$ 
na porcentagem de colonização nas doses mais elevadas.

Nos tratamentos com inoculação de FMA houve incremento de até $88 \%$ no teor de óleos essenciais e $89 \%$ no teor de mentol nos óleos em relação ao tratamento controle, quando não se adicionou fósforo (Tabela 1). Entretanto, não houve diferença significativa entre os tratamentos com inoculação na referida dose de fósforo. Não houve incremento dos teores de óleos essenciais e de mentol nos óleos em função dos FMA, com incremento da adubação fosfatada (Tabela 1).

O teor médio de óleos essenciais foi de $0,80 \mathrm{~g}$ de óleos por $100 \mathrm{~g}$ de matéria fresca da $M$. arvensis, com variações entre 0,49 e $0,94 \mathrm{~g}$ (Tabela 1 ), e o teor médio de mentol foi de $64 \mathrm{~g}$ de mentol por $100 \mathrm{~g}$ de óleos essenciais, com variações entre 38 e $72 \mathrm{~g}$ de mentol (Tabela 1). Kumar et al. (2002) relataram valores de 67 a $77 \mathrm{~g}$ de mentol por $100 \mathrm{~g}$ de óleos essenciais de M. arvensis. Phatak \& Heble (2002) constataram em plantas de $M$. arvensis desenvolvidas no campo, na Índia, teores de $86 \mathrm{~g}$ de mentol por $100 \mathrm{~g}$ de óleos essenciais. Entretanto, experimentos realizados no campo, no Brasil, revelaram teores de $62 \mathrm{~g}$ de mentol por $100 \mathrm{~g} \mathrm{de}$ óleos essenciais (Czepak, 1998).

Nos tratamentos com Glomus clarum, Glomus etunicatum e Acaulospora scrobiculata, não houve alteração significativa nos teores de mentol com o aumento na dose de $\mathrm{P}$, e os valores médios foram de 65 65 e $66 \mathrm{~g}$ de mentol por $100 \mathrm{~g}$ de óleos essenciais, respectivamente (Tabela 1). No tratamento com Gigaspora margarita verificou-se efeito quadrático $\left(\mathrm{Y}=69,8-0,2849 \mathrm{x}-0,00125 \mathrm{x}^{2} ; \mathrm{R}^{2}=0,79\right)$ estimando o menor teor de mentol em 51,3 g por 100 g de óleos essenciais, na dose de $130 \mathrm{~g}$ de fósforo.

As médias encontradas de conteúdo de óleos essenciais de $M$. arvensis variaram de 0,06 a 0,69 g por vaso (Figura 3). Em relação ao conteúdo de mentol, as médias variaram de 0,03 a $0,48 \mathrm{mg}$ por vaso; o menor valor foi verificado no tratamento controle, sem adubação fosfatada e o maior valor foi encontrado no tratamento com 126 e 123 mg de P e inoculação de A. scrobiculata, e a média geral foi de $0,32 \mathrm{~g}$ de mentol por vaso (Figura 4). Na ausência da adubação fosfatada, as maiores produções de óleos essenciais e de mentol foram obtidas nos tratamentos com os fungos Glomus clarum ou Gigaspora margarita, que aumentaram $483 \%$ e $516 \%$ (Figura 3) e 600\% e 733\% (Figura 4), respectivamente, em relação ao tratamento controle.

Poucos são os trabalhos que avaliaram a influência da inoculação de FMA na produção do óleos essenciais em plantas medicinais e, pouco se sabe sobre a influência desses fungos na biossíntese de monoterpenos. Segundo Moreira \& Siqueira (2002), plantas micorrizadas exibem alterações metabólicas, fisiológicas e anatômicas, e vários metabólitos secundários como as giberelinas se acumulam em maior quantidade em plantas com fungos micorrízicos arbusculares. Como as giberelinas são classificadas como diterpenos (Taiz \& Zeiger, 2002), provavelmente plantas micorrizadas possam também acumular monoterpenos, como o mentol.

Foi verificada a influência dos FMA na produção e qualidade dos óleos essenciais de $M$. arvensis, principalmente, nos tratamentos sem adubação fosfatada. Além disso, com incremento de $\mathrm{P}$, o tratamento controle

Tabela 1. Teor de óleos essenciais (g) por $100 \mathrm{~g}$ de matéria fresca e teor de mentol (g) por $100 \mathrm{~g}$ de óleos essenciais de Mentha arvensis, em razão de doses de $\mathrm{P}$ e dos fungos micorrízicos arbusculares ${ }^{(1)}$.

\begin{tabular}{|c|c|c|c|c|c|c|c|c|c|c|}
\hline \multirow[t]{2}{*}{ Fungo } & \multicolumn{5}{|c|}{$\mathrm{P}\left(\mathrm{mg} \mathrm{kg}^{-1}\right.$ de solo $)$} & \multicolumn{5}{|c|}{$\mathrm{P}\left(\mathrm{mg} \mathrm{kg}^{-1}\right.$ de solo $)$} \\
\hline & 0 & 50 & 100 & 200 & Média & 0 & 50 & 100 & 200 & Média \\
\hline & \multicolumn{5}{|c|}{----------- Teor de óleos essenciais ------------ } & \multicolumn{5}{|c|}{------ Teor de mentol nos óleos essenciais ----- } \\
\hline G. margarita & $0,92 \mathrm{a}$ & $0,89 \mathrm{a}$ & $0,76 \mathrm{a}$ & $0,72 \mathrm{a}$ & 0,82 & $67 \mathrm{a}$ & $63 \mathrm{a}$ & $49 b$ & $58 \mathrm{a}$ & 59 \\
\hline G. clarum & $0,86 a$ & $0,83 \mathrm{a}$ & $0,79 \mathrm{a}$ & $0,69 a$ & 0,79 & $60 \mathrm{a}$ & $63 a$ & $66 \mathrm{ab}$ & $69 a$ & 65 \\
\hline G. etunicatum & $0,75 \mathrm{a}$ & $0,76 \mathrm{a}$ & $0,81 \mathrm{a}$ & $0,82 \mathrm{a}$ & 0,78 & $61 \mathrm{a}$ & $62 \mathrm{a}$ & $70 \mathrm{a}$ & $66 a$ & 65 \\
\hline A. scrobiculata & $0,78 \mathrm{a}$ & $0,94 \mathrm{a}$ & $0,85 \mathrm{a}$ & $0,78 \mathrm{a}$ & 0,84 & $72 a$ & $60 \mathrm{a}$ & $69 a$ & $61 \mathrm{a}$ & 66 \\
\hline Sem fungo & $0,49 \mathrm{~b}$ & $0,85 \mathrm{a}$ & $0,79 \mathrm{a}$ & $0,73 \mathrm{a}$ & 0,72 & $38 \mathrm{~b}$ & $71 \mathrm{a}$ & $70 \mathrm{a}$ & $71 \mathrm{a}$ & 63 \\
\hline Média & 0,76 & 0,85 & 0,80 & 0,75 & 0,79 & 60 & 64 & 65 & 65 & 64 \\
\hline
\end{tabular}

(1)Médias seguidas pela mesma letra, na coluna, não diferem entre si pelo Teste de Tukey a 5\%; os coeficientes de variação dos dados de teor de óleos essenciais e teor de mentol foram $14,3 \%$ e $13,7 \%$, respectivamente. 
se iguala aos com inoculação, ou seja, com o incremento de $\mathrm{P}$, houve um incremento no teor de óleos essenciais e no teor de mentol no tratamento controle (Tabela 1). Ichimura et al. (1995) relataram que os teores de linalol, eugenol e 1,8 cineol nos óleos essenciais extraídos de plantas de manjericão (Ocimum basilicum L.) foram maiores com o aumento da adubação fosfatada. Entretanto, estes autores afirmam que os teores de P no solo utilizado no experimento eram baixos, não apresentando os resultados das análises químicas.

Em trabalho realizado no campo com diferentes cultivares de $M$. arvensis e a espécie Glomus fasciculatum em solo com $17 \mathrm{mg} \mathrm{kg}^{-1}$ de P, Gupta et al. (2002) observaram valores diferenciados em relação aos teores de óleos essenciais entre as cultivares; as cultivares Shivalik Gomti e Kalka apresentaram, respectivamente, 0,64, 0,59 e 0,62 g de óleos por $100 \mathrm{~g}$ de matéria fresca. Estes autores também concluíram que a inoculação de FMA proporcionou aumento no conteúdo de óleos essenciais, na produção da matéria fresca e seca da parte aérea, em relação ao tratamento controle.

Kapoor et al. (2002b) observaram incrementos nos teores de óleos essenciais de $90 \%$ em plantas de endro (Anethum graveolens L.) e $72 \%$ em plantas de tomilho indiano (Trachysperum ammi (Linn.) Sprague), com inoculação das espécies Glomus macrocarpum e Glomus fasciculatum, em relação ao controle, em solo estéril com 0,54 mg de P por kg de solo. Neste mesmo trabalho foram constatadas variações nos constituintes químicos dos óleos essenciais em resposta à inoculação de FMA, em que plantas de tomilho indiano com inoculação de Glomus fasciculatum e plantas de endro com inoculação de Glomus macrocarpum apresentaram aumento de $51 \%$ e $116 \%$ nos teores de timol e carvona, em relação ao tratamento controle.

Em plantas de coentro (Coriandrum sativum L.) com inoculação das mesmas espécies de FMA citadas acima, Kapoor et al. (2002a) observaram incrementos de $43 \%$ nos teores de óleos essenciais extraídos dos frutos, e os teores de geraniol e linalol foram maiores em plantas com inoculação em solo estéril e com $0,54 \mathrm{mg}$ de $\mathrm{P}$ por kg de solo. O teor de geraniol foi incrementado com a inoculação de Glomus macrocarpum, e o teor de linalol, com a inoculação de Glomus fasciculatum. Os constituintes dos óleos essenciais avaliados são como o mentol, monoterpenos (Mann, 2001).
Tanto a inoculação de FMA como a adubação fosfatada influenciaram a quantidade e a qualidade dos óleos essenciais de $M$. arvensis, demonstrando a necessidade do desenvolvimento de trabalhos cujo objetivo seja compreender os mecanismos pelos quais esses fungos interferem na produção e qualidade dos óleos essenciais.

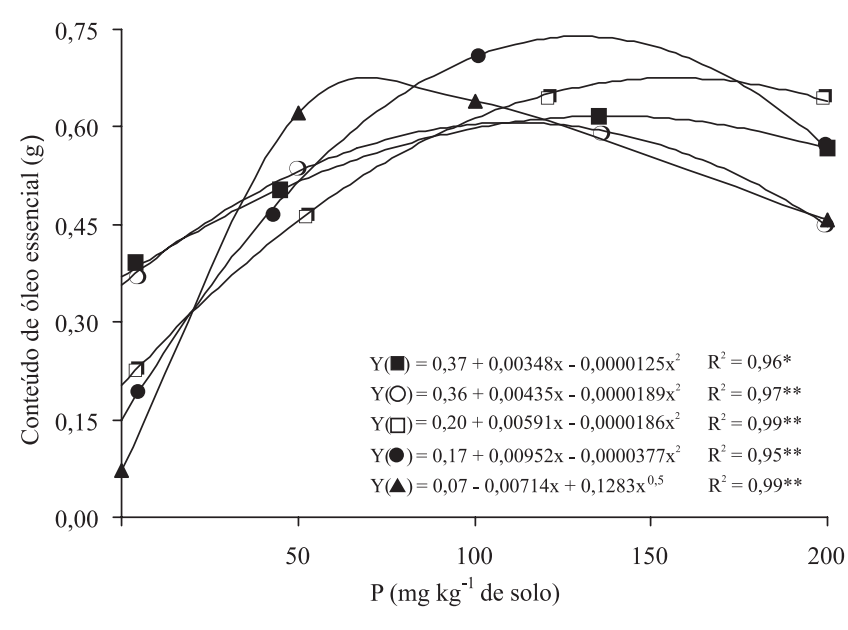

Figura 3. Conteúdo de óleos essenciais, por vaso, na matéria fresca da Mentha arvensis L. em razão das doses de P e dos fungos micorrízicos arbusculares Gigaspora margarita ( Glomus clarum (○), Glomus etunicatum ( $\square$ ), Acaulospora scrobiculata $(\bullet)$ e controle $(\boldsymbol{\Lambda})$

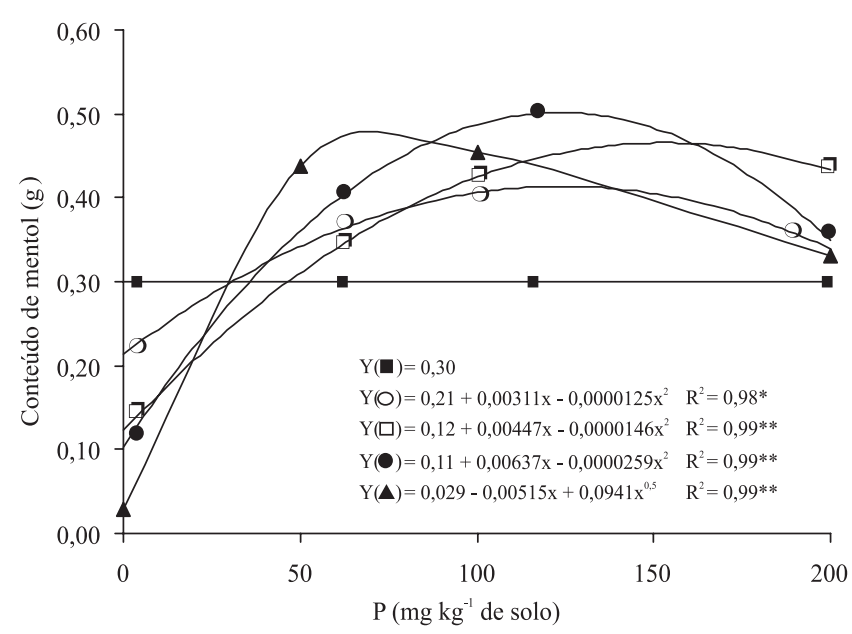

Figura 4. Conteúdo de mentol por vaso em razão das doses de $\mathrm{P}$ e dos fungos micorrízicos arbusculares, Gigaspora margarita $(\mathbf{\square})$, Glomus clarum $(\bigcirc)$, Glomus etunicatum $(\square)$, Acaulospora scrobiculata $(\bullet)$ e controle $(\mathbf{\Lambda})$ 


\section{Conclusões}

1. A inoculação de fungos micorrízicos arbusculares (FMA) e as doses de P influenciam o crescimento, os teores e a composição dos óleos essenciais das plantas de Mentha arvensis L.

2. Na ausência da adubação fosfatada, os FMA são benéficos para produção de matéria fresca, produção e qualidade dos óleos essenciais.

3. Nos tratamentos micorrizados, com a aplicação de adubação fosfatada, verifica-se tendência de redução dos teores e da qualidade dos óleos essenciais em Mentha arvensis L.

\section{Agradecimentos}

Ao Conselho Nacional de Desenvolvimento Científico e Tecnológico, pela concessão de bolsa aos autores.

\section{Referências}

BRESSAN, W.; SIQUEIRA, J.O.; VASCONCELLOS, C.A.; PURCINO, A.C.P. Fungos micorrízicos e fósforo, no crescimento, nos teores de nutrientes e na produção do sorgo e soja consorciados. Pesquisa Agropecuária Brasileira, v.36, p.315-323, 2001.

BRESSAN, W.; VASCONCELLOS, C.A. Alterações morfológicas no sistema radicular do milho induzidas por fungos micorrízicos e fósforo. Pesquisa Agropecuária Brasileira, v.37, p.509-517, 2002 COSTA, C.M.C.; MAIA, L.C.; CAVALCANTE, U.M.T.; NOGUEIRA, R.J.M.C. Influência de fungos micorrízicos arbusculares sobre o crescimento de dois genótipos de aceroleira (Malpighia emarginata D.C.). Pesquisa Agropecuária Brasileira v.36, p.893-901, 2001.

CZEPAK, M.P. Produção de óleo bruto e mentol cristalizável em oito freqüências de colheita de menta (Mentha arvensis L.) In: MING L.C. (Coord.). Plantas medicinais aromáticas e condimentares: avanços na pesquisa agronômica. Botucatu: Unesp, 1998. v.2, p.52-80.

FARIA, A.Y.K.; MATSUOKA, M.; OLIVEIRA, E.A. de S ; LOUREIRO, M. de F. Fungos micorrízicos arbusculares em sucupirapreta: efeito de substratos sobre a colonização. Horticultura Brasileira, v.18, p.913-914, 2000.

GIOVANNETTI, M.; MOSSE, B. An evaluation of techniques for measuring vesicular-arbuscular mycorrhizal infection in roots. New Phytologist, v.84, p.489-500, 1980.

GRACE, C.; STRIBLEY, P. A safer procedure for routine staining of vesicular-arbuscular mycorrhizal fungi. Mycological Research, v.95, p.1160-1162, 1991

GROTKASS, C.; HUTTER, I.; FELDMANN, F. Use of arbuscular mycorrhizal fungi to reduce weaning stress of micropropagated
Baptisia tinctoria (L.) R.BR. Acta Horticulturae, n.530, p.305311, 2000 .

GUPTA, M.L.; PRASAD, A.; RAM, M.; KUMAR, S. Effect of the vesicular-arbuscular mycorrhizal (VAM) fungus Glomus fasciculatum on the essential oil yield related characters and nutrient acquisiton in the crops of different cultivars of menthol mint (Mentha arvensis) under field conditions. Bioresource Technology, v.81, p.77-79, 2002.

ICHIMURA, M.; IKUSHIMA, M.; MIYAZAKI, T.; KIMURA, M. Effect of phosphorus on growth and concentration of mineral elements and essential oils of sweet basil leaves. Acta Horticulturae, n.396, p.195-202, 1995.

JACKSON, L.E.; MILLER, D.; SMITH, S.E. Arbuscular mycorrhizal colonization and growth of wild and cultivated lettuce in response to nitrogen and phosphorus. Scientia Horticulturae, v.94, p.205-218, 2002

KAPOOR, R.; GIRI, B.; MUKERJI, K.G. Glomus macrocarpum: a potential bioinoculant to improve essential oil quality and concentration in Dill (Anethum graveolens L.) and Carum (Trachyspermum ammi (Linn.) Sprague). World Journal of Microbiology and Biotechnology, v.18, p.459-463, 2002a.

KAPOOR, R.; GIRI, B.; MUKERJI, K.G. Mycorrhization of coriander (Coriandrum sativum L.) to enhance the concentration and quality of essential oil. Journal of the Science of Food and Agriculture, v.82, p.339-342, 2002b.

KUMAR, S.; BAHL, J.R.; BANSAL, R.P.; GUPTA, A.K.; SINGH, V.; SHARMA, S. High economic returns from companion and relay cropping of bread wheat and menthol mint in the winter-summer season in north Indian plains. Industrial Crops and Products, v.15, p.103-114, 2002.

MAIA, N.B. Efeito da nutrição mineral na qualidade do óleo essencial da menta (Mentha arvensis L.) cultivada em solução nutritiva. In: MING, L.C. (Coord.). Plantas medicinais aromáticas e condimentares: avanços na pesquisa agronômica. Botucatu: Unesp, 1998. v.2, p.81-95.

MANN, J. Secondary metabolism. 2nd ed. Oxford: Oxford Science, 2001. 374p

MARTINS, M.A.; GONÇALVES, G.F. de; SOARES, A.C.F. Efeito de fungos micorrízicos arbusculares associados a compostos fenólicos, no crescimento de mudas de mamoeiro. Pesquisa Agropecuária Brasileira, v.35, p.1465-1471, 2000.

MATOS, F.J. de A. Plantas medicinais: guia de seleção e emprego de plantas usadas em fitoterapia no Nordeste do Brasil. 2.ed. Fortaleza: Edições UFC, 2000. 346p.

MOREIRA, F.M. de S.; SIQUEIRA, J.O. Microbiologia e bioquímica do solo. Lavras: Ufla, 2002. 626p.

NOGUEIRA, M.A.; CARDOSO, E.J.B.N. Produção de micélio externo por fungos micorrízicos arbusculares e crescimento da soja 
em função de doses de fósforo. Revista Brasileira de Ciência do Solo, v.24, p.329-338, 2000

PHATAK, S.V.; HEBLE, M.R. Organogenesis and terpenoi synthesis in Mentha arvensis. Fitoterapia, v.73, p.32-39, 2002.

PRALON, A.Z.; MARTINS, M.A. Utilização do resíduo industria ferkal na produção de mudas de Mimosa caesalpiniafolia, em estéril de extrato de argila, inoculadas com fungos micorrízicos arbusculares rizóbio. Revista Brasileira de Ciência do Solo, v.25, p.55-63, 2001.

SAMARÃO, S.S.; MARTINS, M.A. Influência de fungos micorrízicos arbusculares, associada à aplicação de rutina, no crescimento de mudas de goiabeira (Psidium guajava L.). Revista Brasileira de Fruticultura, v.21, p. 196-199, 1999.
SMITH, S.E.; READ, D.J. Mycorrhizal symbiosis. 2nd ed. San Diego: Academic Press, 1997. 605p.

SOARES, A.C.F.; MARTINS, M.A. Influência de fungos micorrízicos arbusculares, associada à adição de compostos fenólicos, no crescimento de mudas de maracujazeiro amarelo (Passiflora edulis f. flavicarpa). Revista Brasileira de Ciência do Solo, v.24, p.731740, 2000.

TAIZ, L.; ZEIGER, E. Plant physiology. Redwood City: Benjamin/ Cummings, 2002. 630p

VOLPIN, H.; ELKIND, Y.; OKON, Y.; KAPULNIK, Y. A vesicular arbuscular mycorrhizal fungus (Glomus intraradix) induces a defense response in alfafa roots. Plant Physiology, v.104, p.683-689, 1994.

Recebido em 4 de junho de 2003 e aprovado em 23 de junho de 2004 